\title{
FRACTIONAL INTEGRATION OPERATOR OF VARIABLE ORDER IN THE HOLDER SPACES $H^{\lambda(x)}$
}

\author{
BERTRAM ROSS \\ University of New Haven \\ 300 Orange Avenue, West Haven, CT 06516, USA \\ and \\ STEFAN SAMKO \\ Rostov State University \\ 105, Bol'shaya Sadovaya, Rostov-on-Don, 344711, Russia \\ Currently Fulbright Scholar at the University of New Haven, USA
}

(Received June 17, 1993 and in revised form September 20, 1993)

\begin{abstract}
The fractional integrals $I_{a_{+}}^{\alpha(x)} \varphi$ of variable order $\alpha(x)$ are considered. A theorem on mapping properties of $I_{a+}^{\alpha(x)}$ in Holder-type spaces $H^{\lambda(x)}$ is proved, this being a generalization of the well known Hardy-Littlewood theorem.
\end{abstract}

KEYWORDS: Fractional integration, variable fractional order, mapping properties, Holder continuous functions, Hardy-Littlewood theorem.

1991 AMS SUBJECT CLASSIFICATION CODES. 26 A33, 26 A16

\section{INTRODUCTION}

In the paper [1] the authors introduced and investigated the fractional integrals

$$
I_{a+}^{\alpha(x)} \varphi=\frac{1}{\Gamma[\alpha(x)]} \int_{a}^{x} \varphi(t)(x-t)^{\alpha(x)-1} d t
$$

of variable order $\alpha(x)>0$, and considered the corresponding versions of fractional differentiation as well.

In this paper we prove the theorem on the behaviour of the operator $I_{a+}^{\alpha(x)}$ in the generalized Holder spaces $H^{\lambda(x)}$ the order of which also 
depends on the point $x$. This is a generalization of the Hardy - Littlewood theorem, well - known in the case of constant orders $\alpha(x)=\alpha=$ const and $\lambda(x)=\lambda=$ const (Hardy and Littlewood [2]; see also Samko et al [3], p. 53-54). our interest in integration and differentiation of a variable order is motivated not only by the desire to generalize the classical notion, but by some far reaching goals as well. There is the well known theory of fractional Sobolev type spaces, see its elements e.g. in [3], sections 26-27. These spaces consist of functions whose smoothness property can be expressed either globally or locally in terms of the existence of fractional derivatives. The smoothness property of a function may, however, vary from point to point. The construction of the corresponding sobolev type spaces is an open question. The notion introduced in (1) is the appropriate tool for this purpose. In this paper we deal only with the question of improving the smoothness property, expressed in terms of the Holder type condition, by the operator (1), and the theorem proved may be considered as a starting point for further investigations of functions with varying order of smoothness. In Section 1 we give all required definitions and some auxilliary lemmas, while Section 2 contains the statement and the proof of the main result.

In what follows the letter $c$ may denote different positive constants.

\section{PRELIMINARIES}

Let $\Omega=[a, b],-\infty<a<b<\infty$. The following is a generalization of the Holder space $H^{\lambda}, 0<\lambda \leq 1$.

DEFINITION 1. We say that $f(x) \in H^{\lambda(x)}(\Omega)$ where $\lambda(x)$ is a positive (not necessarily continuous) function, $0<\lambda(x) \leq 1$, if

$$
|f(x+h)-f(x)| \leq c|h|^{\lambda(x)}
$$

for all $x, x+h \in[a, b]$.

It is easily seen that (2) implies that

$$
|f(x+h)-f(x)| \leq c|h|^{\lambda(x+h)}
$$

So, it is not difficult to show that the definition of the class $H^{\lambda(x)}(\Omega)$ by (2) is equivalent to the definition by means of the following symmetrical 
inequality

$$
\left|f\left(x_{1}\right)-f\left(x_{2}\right)\right| \leq c\left|x_{1}-x_{2}\right|^{\max \left\{\lambda\left(x_{1}\right), \lambda\left(x_{2}\right)\right\}}
$$

It is easily seen that $H^{\lambda(x)}(\Omega)$ is a ring with respect to the usual multiplication. It is a Banach space with respect to the norm

$$
\begin{aligned}
& \|f\|_{H^{\lambda(x)}}=\sup _{x \in \Omega} \sup _{\substack{|h| \leq 1 \\
h+x \in S}} \frac{|f(x+h)-f(x)|}{|h|^{\lambda(x)}} \simeq \\
& \sup _{x_{1}, x_{2} \in \Omega} \frac{\left|f\left(x_{1}\right)-f\left(x_{2}\right)\right|}{\left|x_{1}-x_{2}\right|^{\max \left\{\lambda\left(x_{1}\right), \lambda\left(x_{2}\right) \mid\right.}}
\end{aligned}
$$

where $\simeq$ denotes the equivalence: $f \simeq g \Leftrightarrow c_{1} f \leq g \leq c_{2} f, c_{1}>0, c_{2}>0$.

Generalizing Definition 1 we give the following

DEFINITION 2. We say that $f(x) \in H^{\lambda(x), \mu(x)}(\Omega)$ where $\lambda(x)$ and $\mu(x)$ are given functions, $0<\lambda(x) \leq 1,-x<\mu(x)<\infty$, if

$$
|f(x+h)-f(x)| \leq c|h|^{\lambda(x)}\left(\ln \frac{1}{|h|}\right)^{\mu(x)},|h|<\frac{1}{2},
$$

under assumption that $x, x+h \in \Omega$.

We shall need the following auxilliary assertions.

LEMMA 1. Let the function $\lambda(x) \in C(\Omega)$ satisfy the condition

$$
|\lambda(x+h)-\lambda(x)| \leq \frac{A}{1+\ln \frac{b-a}{h}}, A=\text { const }>0
$$

for all $x, x+h \in \Omega$. Then the function

$$
g_{s, t}(x)=(x-a)^{\lambda[t+s(x-a)]-\lambda(t)}
$$

where $x, t, t+s(x-a) \in \Omega, s \in[0,1]$, is bounded from zero and infinity:

$$
0<d^{-A} \leq g_{s, t}(x) \leq d^{A}<x
$$


PROOF. Since

$\ln g_{s, t}(x)=\{\lambda[t+s(x-a)]-\lambda(t)\} \ln (x-a)$, by (5) we have

$$
\left|\ln g_{s, t}(x)\right| \leq \frac{A|\ln (x-a)|}{1+\ln \frac{b-a}{s(x-a)}} \leq \frac{A|\ln (x-a)|}{1+\ln \frac{b-a}{x-a}}
$$

Simple calculations show that the maximum of the right-hand side is $A$ $\max \{1,|\ln (b-a)|\}$. Really, let $f(y)=A|y|(1+c-y)^{-1}$, where $-x y \leq c=$ $\ln (b-a)$. Suppose $b-a \geq 1$ first. For $y>0$ we have $f(y)=A y(1+c-y)^{-1} \leq A y \leq$ $A \ln (b-a)$. If $y<0$, then $f(y)=A|y|(1+c+|y|)^{-1} \leq A$. Therefore,

$$
f(y) \leq A \max \{1, \ln (b-a)\}
$$

in the case $c=\ln (b-a)>0$. Let now $c<0$. Then $y<0$ and $f(y)=A-$ $A(1+c)(1+c-y)^{-1}$ if $1+c \geq 0$. If $1+c<0$, we have $f^{\prime}(y)=-A(1+c)(1+c-y)^{-2}$ $>0$, so that $f(y) \leq f(c)$ for $y \leq c$ which gives $f(y) \leq A|c|=A|\ln (b-a)|$. So,

$$
f(y) \leq A \max \{1,|\ln (b-a)|\}
$$

in all cases. Therefore, $\left|\ln { }_{s, t} g(x)\right| \leq A \max \{1,|\ln (b-a)|\}$, whence (6) follows. LEMMA 2. For any function $\alpha(x)$ such that $0 \leq \alpha(x) \leq 1$, the inequality

$$
\left|(x+h)^{\alpha(x)}-x^{\alpha(x)}\right| \leq h^{\alpha(x)}, h>0, x>0
$$

holds.

PROOF. By dividing all members in $(8)$ by $h^{\alpha(x)}$, we see that the inequality (8) is equivalent to the inequality $f(\zeta) \leq 1$ with $f(\zeta)=$ $(1+\zeta)^{\alpha(x)}-\zeta^{\alpha(x)}>0$ for all $\zeta>0$, which is evident because $f^{\prime}(\zeta) \leq 0$ and $f(0)=1$.

LEMMA 3. Let $0<\alpha \leq 1$ and $-\alpha<\lambda<1-\alpha$. Then

$$
\int_{0}^{\alpha} t^{\lambda}\left[t^{(x-1}-(t+1)^{(x-1}\right] d t=\frac{\sin \alpha \pi}{\sin (\alpha+\lambda) \pi} B(\alpha, 1+\lambda)
$$


PROOF. Let $A(\lambda, \alpha)$ denote the left-hand side in (9). After the substitution $t+1=s^{-1}$ we have

$$
A(\lambda, \alpha)=\int_{0}^{1}(1-s)^{\lambda} \frac{(1-s)^{\alpha-1}-1}{s^{1+\lambda+\alpha}} d s
$$

Hence

$$
A(\lambda, \alpha)=\int_{0}^{1}(1-s)^{\lambda} \frac{(1-s)^{\alpha-1}+(1-s)^{\mu}}{s^{1+\lambda+\alpha}} d s+\int_{0}^{1}(1-s)^{\lambda} \frac{(1-s)^{\mu}-1}{s^{1+\lambda+\alpha}} d s
$$

with $\mu$ not determined as yet. Hence, by (10)

$$
A(\lambda, \alpha)=A(\lambda+\mu, \alpha-\mu)+\int_{0}^{1}(1-s)^{\lambda} \frac{(1-s)^{\mu}-1}{s^{1+\lambda+\alpha}} d s
$$

The second integral here is evaluated by means of analytical continuation with respect to $\mu$ :

$$
\int_{0}^{1}(1-s)^{\lambda} \frac{(1-s)^{\mu-1}}{s^{1+\lambda+\alpha}} d s=B(\lambda+\mu+1,-\lambda-\alpha)-B(\lambda+1,-\lambda-\alpha)
$$

under the appropriate conditions on the parameters $\mu$ and $\lambda$. So,

$$
A(\lambda, \alpha)=A(\lambda+\mu, \alpha-\mu)+\Gamma(-\lambda-\alpha)\left[\frac{\Gamma(\lambda+\mu+1)}{\Gamma(\mu+1-\alpha)}-\frac{\Gamma(\lambda+1)}{\Gamma(1-\alpha)}\right]
$$

Simple calculations show that $A(0, \alpha)=1 / \alpha$. So, choosing $\mu=-\lambda$ we have

$$
A(\lambda, \alpha)=\frac{1}{\lambda+\alpha}+\Gamma(-\lambda-\alpha)\left[\frac{1}{\Gamma(1-\alpha-\lambda)}-\frac{\Gamma(\lambda+1)}{\Gamma(1-\alpha)}\right]
$$

which gives (9) after easy calculations.

\section{THE MAIN THEOREM}

Considering the fractional integral $I_{a_{+}}^{\alpha(x)} \varphi$, defined in (1), of the function $\varphi(x) \in H^{\lambda(x)}$, we shall assume the following conditions on $\alpha(x)$ and $\lambda(x)$ to be satisfied: 
i) $0<\alpha(x) \leq 1$ with $m=\inf _{x \in \Omega} \alpha(x)>0$ and $\quad \alpha(x) \in H^{\mu(x)}(\Omega), 0<\delta \leq \mu(x) \leq 1$;

ii) $0<\lambda(x) \leq 1$ and $\lambda(x)$ satisfies the condition (5); iii) $\alpha(x)+\lambda(x) \leq 1$.

THEOREM. Let the conditions $i)-i i i)$ be satisfied and let $\varphi(x) \in$ $H^{\lambda(x)}(\Omega)$. Then $I_{a+}^{x(x)} \varphi$ has the form

$$
I_{a+}^{\alpha(x)} \varphi=\frac{\varphi(a)}{\Gamma[1+(x(x)]}(x-a)^{\alpha(x)}+f(x)
$$

where

$$
f(x) \in H^{\gamma(x)}(\Omega), \gamma(x)=\min \{\lambda(x)+(x(x), \mu(x)\}
$$

$$
f(x) \in H^{\gamma(x), 1}(\Omega)
$$
if $\max _{x \in \Omega}[\lambda(x)+\alpha(x)]=1$. In both cases

$$
|f(x)| \leq c(x-a)^{\lambda(x)+\alpha(x)} \leq c(x-a)^{\lambda(a)+\alpha(a)}
$$

REMARK. The assertion (13) can be exactified:

$$
|f(x+h)-f(x)| \leq \frac{c}{1-\alpha(x)-\lambda(x)}|h|^{\gamma(x)}
$$

for all $x \in \Omega$ such that $\alpha(x)+\lambda(x)<1$ and

$$
|f(x+h)-f(x)| \leq c|h|^{\gamma(x)} \text { In } \frac{1}{|h|},|h|<1
$$

for those $x$ which give the equality $\alpha(x)+\lambda(x)=1$, c being a positive constant not depending on $x$ and $h$ (see the proof of the theorem).

PROOF OF THE THEOREM. From the conditions i) and ii) it follows that $\gamma(x)=: \frac{1}{\Gamma[\alpha(x)]} \in H^{\mu(x)}(\Omega)$. Really, $|\gamma(x+h)-\gamma(x)| \leq|\alpha(x+h)-\alpha(x)|$ 
$\max _{a S x \leq b}\left(\left|\Gamma^{\prime}(x) / \Gamma(x)\right|\right) \leq c|h|^{\mu(x)}$. So, it is sufficient to consider the integral

$$
g(x)=\int_{a}^{x}(x-t)^{(x(x)-1} \varphi(t) d t
$$

we have to prove that

$$
g(x)=\frac{\varphi(a)}{\alpha(x)}(x-a)^{\alpha(x)}+f_{0}(x)
$$

where

$$
f_{0}(x) \in H^{\lambda(x)+\alpha(x)} \text { or } f_{0}(x) \in H^{\lambda(x)+\alpha(x), 1}
$$

if $\max [\lambda(x)+\alpha(x)]<1$ or $\max [\lambda(x)+\alpha(x)]=1$, respectively (with the exactification (15)-(16), if we will). The derivation itself of the equality (17) with the function

$$
f_{0}(x)=\int_{a}^{x} \frac{\varphi(t)-\varphi(a)}{(x-t)^{1-\alpha(x)}} d t
$$

is obvious. For the function $f_{0}(x)$ we prove first the estimate (14). We have

$$
\begin{aligned}
& \quad\left|f_{0}(x)\right| \leq\|\varphi\|_{H} \lambda(x) \int_{a}^{x}(x-t)^{\alpha(x)-1}(t-a)^{\lambda(t)} d t \\
& =c(x-a)^{\alpha(x)+\lambda(a)} \int_{0}^{1}(1-s)^{\alpha(x)-1} s^{\lambda[a+s(x-a)]} g_{s, a}(x) d s
\end{aligned}
$$

where $g_{s, a}(x)$ - is the function (6). By lemma 1 we have

$$
\left|f_{0}(x)\right| \leq c_{1}(x-a)^{\alpha(x)+\lambda(a)} \int_{0}^{1}(1-s)^{m-1} d s=c_{2}(x-a)^{\alpha(x)+\lambda(a)}
$$

Hence, to obtain the estimate (14), it remains to observe that

$$
(x-a)^{\alpha(x)+\lambda(a)} \leq c(x-a)^{\alpha(x)+\lambda(x)} \leq c_{1}(x-a)^{\alpha(a)+\lambda(a)} \leq c_{2}(x-a)^{\alpha(x)+\lambda(x)}
$$

which follows from the lemma 1 (we remark that the condition (5) for 
$\lambda(x)+\alpha(x)$ is fulfilled because it is satisfied for $\lambda(x)$ by the assumption in $i i)$ and for $\alpha(x)$ by the assumption in $i)$ ). Thus, (14) is proved.

To prove the statements (18) we consider the difference $f_{0}(x+h)-f_{0}(x)$ taking $h$ positive. (If $h<0$, by denoting $x+h=x_{1}, x_{1}=x_{1}+(-h)$ we reduce the consideration to the case of positive increment). We represent this difference as

$$
\begin{aligned}
& f_{0}(x+h)-f_{0}(x)=\int_{-h}^{x-a} \frac{\varphi_{0}(x-t) d t}{(t+h)^{1-\alpha(x+h)}}-\int_{0}^{x-a} \frac{\varphi_{0}(x-t) d t}{t^{1-\alpha(x)}} \\
& =\int_{-h}^{x-a} \varphi_{0}(x-t)\left[(t+h)^{\alpha(x+h)-1}-(t+h)^{\alpha(x)-1}\right] d t \\
& \left.+\iint_{-h}^{x-a} \frac{\varphi_{0}(x-t) d t}{(t+h)^{1-\alpha(x)}}-\int_{0}^{x-a} \frac{\varphi_{0}(x-t) d t}{t^{1-(x(x)}}\right]=J_{0}+J,
\end{aligned}
$$

with $\varphi_{0}(t)=\varphi(t)-\varphi(a)$. We estimate $J_{0}$ first. We have

$$
\begin{aligned}
\left|J_{0}\right| \leq \max \left|\varphi_{0}(t)\right| \int_{-h}^{x-a}\left|(t+h)^{\alpha(x+h)-1}-(t+h)^{\alpha(x)-1}\right| d t \\
=c \int_{0}^{x+h-a}\left|t^{\alpha(x+h)-1}-t^{\alpha(x)-1}\right| d t
\end{aligned}
$$

Since $t^{u}-t^{v}=t^{\xi} \ln t(u-v)$ with $\xi$ between $u$ and $v$, we obtain

$$
\left|J_{0}\right| \leq c|\alpha(x+h)-\alpha(x)| \int_{0}^{x+h-a} t^{F-1}|\ln t| d t
$$

with $\xi$ between $\alpha(x+h)$ and $\alpha(x)$ so that $\xi \geq m>0$. Since $t^{\xi-1} \leq A t^{m-1}$ with $A=\max \left\{1,(b-a)^{1-m}\right\}$, we have 


$$
\left|J_{0}\right| \leq \operatorname{cAh}^{\mu(x)} \int_{0}^{2(b-a)} t^{m-1}|\ln t| d t=c_{1} h^{\mu(x)}
$$

As regards the term $J$ in (19) it should be decomposed similarly to the proof of the Hardy - Littlewood theorem for the case $\alpha(x)=\alpha=$ const, $\lambda(x)$ $=\lambda=$ const, see Samko et al [3]. We have

$$
J=J_{1}+J_{2}+J_{3}
$$

with

$$
\begin{gathered}
J_{1}=\varphi_{0}(x) \int_{x-a}^{x-a+h}(t+h)^{\alpha(x)-1} d t, J_{2}=\int_{-h}^{0} \frac{\varphi_{0}(x-t)-\varphi_{0}(x)}{(t+h)^{1-\alpha(x)}} d t, \\
J_{3}=\int_{0}^{x-a}\left[(t+h)^{\alpha(x)-1}-t^{\alpha(x)-1}\right]\left[\varphi_{0}(x-t)-\varphi_{0}(x)\right] d t .
\end{gathered}
$$

The estimate of $J_{1}$. In the case $h \leq x-a$, for the term

$$
J_{1}=\frac{\varphi_{0}(x)}{\alpha(x)}\left[(x-a+h)^{\alpha(x)}-(x-a)^{\alpha(x)}\right]
$$

we use the inequality

$$
\left|\varphi_{0}(x)\right| \leq\|\varphi\|_{H^{\lambda(x)}}(x-a)^{\lambda(x)}
$$

and the inequality $(1+t)^{\beta}-1 \leq \beta t$, with $0 \leq \beta \leq 1$ and $t>0$. We have

$$
\begin{aligned}
& \left|J_{1}\right| \leq c \frac{(x-a)^{\lambda(x)+\alpha(x)}}{\alpha(x)}\left|\left(1+\frac{h}{x-a}\right)^{\alpha(x)}-1\right| \\
& \leq c(x-a)^{\lambda(x)+\alpha(x)} \frac{h}{x-a}=c(x-a)^{\lambda(x)+\alpha(x)-1} h \leq c h^{\lambda(x)+\alpha(x)} .
\end{aligned}
$$


If $x-a \leq h$ we use (21) again and have

$$
\left|J_{1}\right| \leq c \frac{(x-a)^{\lambda(x)}}{\alpha(x)}\left|(x+h-a)^{\alpha(x)}-(x-a)^{\alpha(x)}\right| \text {. }
$$

Hence, by ( 8 )

$$
\left|J_{1}\right| \leq \frac{c}{m}(x-a)^{\lambda(x)} h^{\alpha(x)} \leq \frac{c}{m} h^{\lambda(x)+\alpha(x)} .
$$

The estimate of $J_{2}$. The estimate of $J_{2}$ is completely like in the case of constant order:

$$
\left|J_{2}\right| \leq c \int_{-h}^{0} \frac{|t|^{\lambda(x)} d t}{(t+h)^{1-\alpha(x)}}=c_{1} h^{\lambda(x)+\alpha(x)}
$$

The estimate of $J_{3}$. We have

$$
\begin{aligned}
& \left|J_{3}\right| \leq c \int_{0}^{x-a} t^{\lambda(x)}\left|(t+h)^{\alpha(x)-1}-t^{\alpha(x)-1}\right| d t \\
& =c h^{\lambda(x)+\alpha(x)} \int_{0}^{\frac{x-a}{h}} t^{\lambda(x)}\left[t^{\alpha(x)-1}-(t+1)^{\alpha(x)-1}\right] d t
\end{aligned}
$$

If $x-a \leq h$, we have

$$
\begin{aligned}
\left|J_{3}\right| & \leq c h^{\lambda(x)+(x(x)} \int_{0}^{1} t^{\lambda(x)}\left[t^{\alpha(x)-1}+(t+1)^{\alpha(x)-1}\right] d t \\
& \leq c h^{\lambda(x)+\alpha(x)} \int_{0}^{1}\left(t^{m-1}+1\right) d t=c h^{\lambda(x)+\alpha(x)}
\end{aligned}
$$

Let $x-a \geq h$. Then 


$$
\left|J_{3}\right| \leq c h^{\lambda(x)+\alpha(x)} \int_{0}^{\alpha} t^{\lambda(x)}\left[t^{\alpha(x)-1}-(t+1)^{\alpha(x)-1}\right] d t
$$

under the assumption that $\lambda(x)+\alpha(x)<1$. Then by the lemma 3 we have

$$
\left|J_{3}\right| \leq c h^{\lambda(x)+\alpha(x)} \frac{\sin [\alpha(x) \pi]}{\sin [\alpha(x) \pi+\lambda(x) \pi]} \text {. }
$$

because $B(\alpha(x), 1+\lambda(x)) \leq$ const. Since $1 /(\sin \pi t) \leq c(1-t)^{-1}$, we have

$$
\left|J_{3}\right| \leq \frac{c_{1} h^{\lambda(x)+\alpha(x)}}{1-\alpha(x)-\lambda(x)}
$$

If $\lambda(x)+\alpha(x)=1$, we split the integration in (24) from 0 to 1 and from 1 to $(x-a) / h$ and havehave from $(24)$

$$
\begin{gathered}
\qquad J_{3} \mid \leq c_{2} h\left[1+\int_{1}^{\frac{x-a}{h}} t^{-1} d t\right] \\
\text { since }\left|t^{\alpha(x)-1}-(t+1)^{\alpha(x)-1}\right| \leq c t^{\alpha(x)-2} \text { for } t>1 \text {. So, } \\
\left|J_{3}\right| \leq c h\left[1+\ln \frac{x-a}{h}\right] \leq c_{1} h \ln \frac{1}{h}
\end{gathered}
$$

because $x-a \geq h$.

Gathering the estimates (20), (22), (23), (24), (25) and (26) we obtain the inequalities (15) - (16) which proves the theorem.

ACKNOWLEDGEMENT. This work was partially funded by a Fulbright grant.

The authors are thankful to the referee for a careful reading of the manuscript and his suggestions. 


\section{REFERENCES}

1 ROSS,B. AND SAMKO,S. Integration and differentiation to a variable fractional order, Integral Transforms and Special Functions, $1(1993)$, N 3 .

2. HARDY,G.H. AND LITTLEWOOD, J.E. SOme properties of fractional integrals, I, Math. Zeit., 27(1928), N 4, 565-606.

3. SAMKO,S.,KILBAS A. AND MARICHEV,O. Integrals and Derivatives of Fractional Order. Theory and Applications. Gordon \& Breach Sci. Publ., 1993 (to appear). (Russian edition by "Nauka i Tekhnika", Minsk, 1987). 


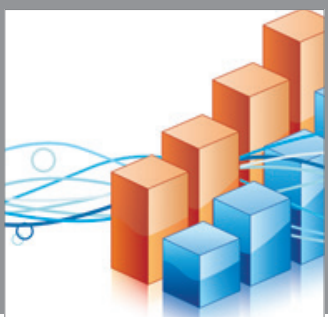

Advances in

Operations Research

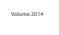

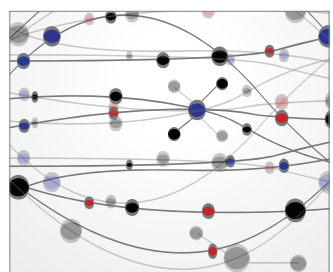

\section{The Scientific} World Journal
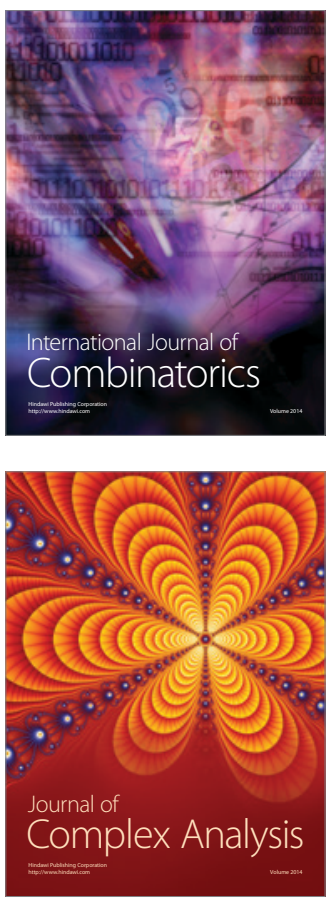

International Journal of

Mathematics and

Mathematical

Sciences
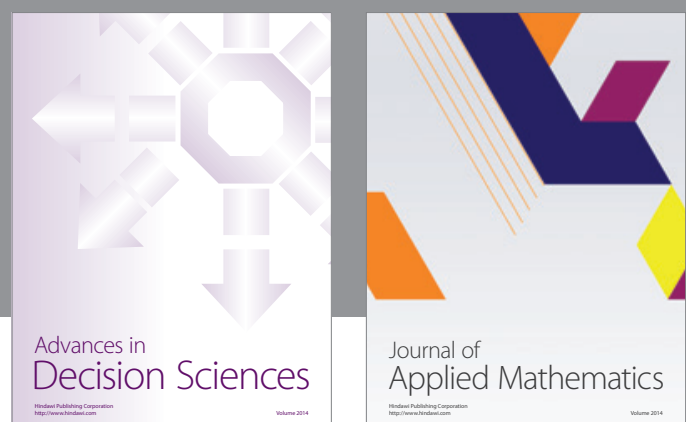

Journal of

Applied Mathematics
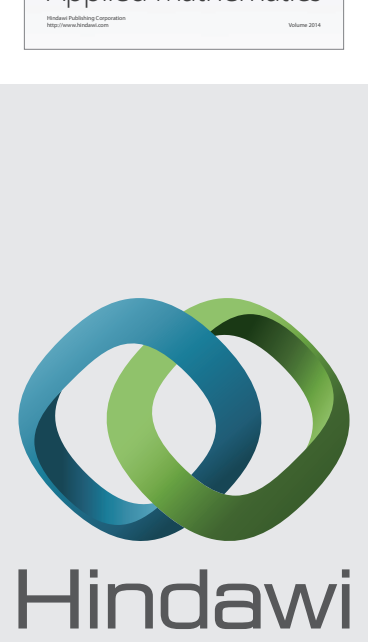

Submit your manuscripts at http://www.hindawi.com
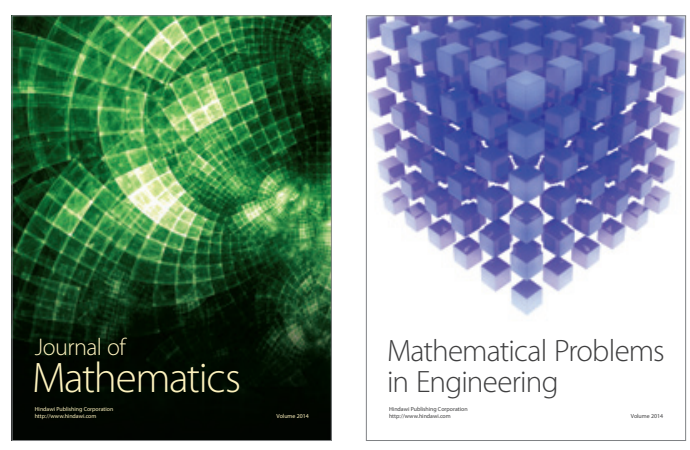

Mathematical Problems in Engineering
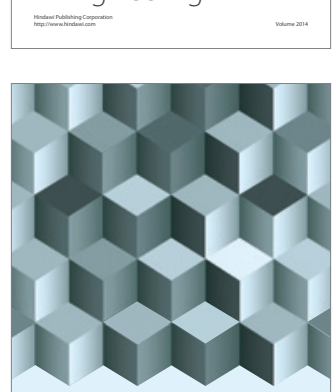

Journal of

Function Spaces
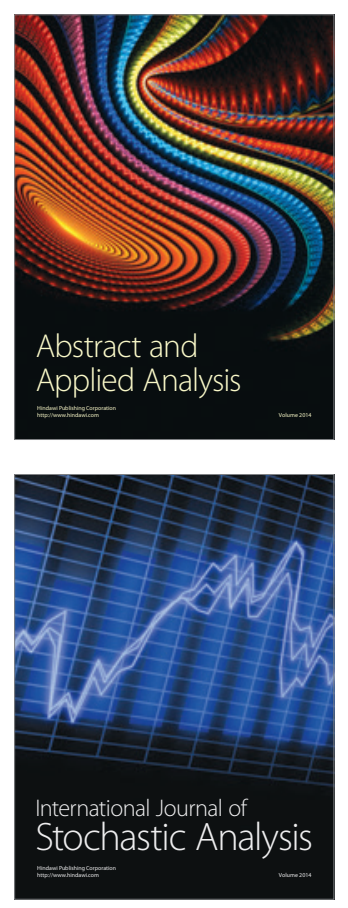

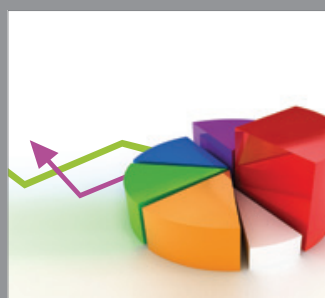

ournal of

Probability and Statistics

Promensencen
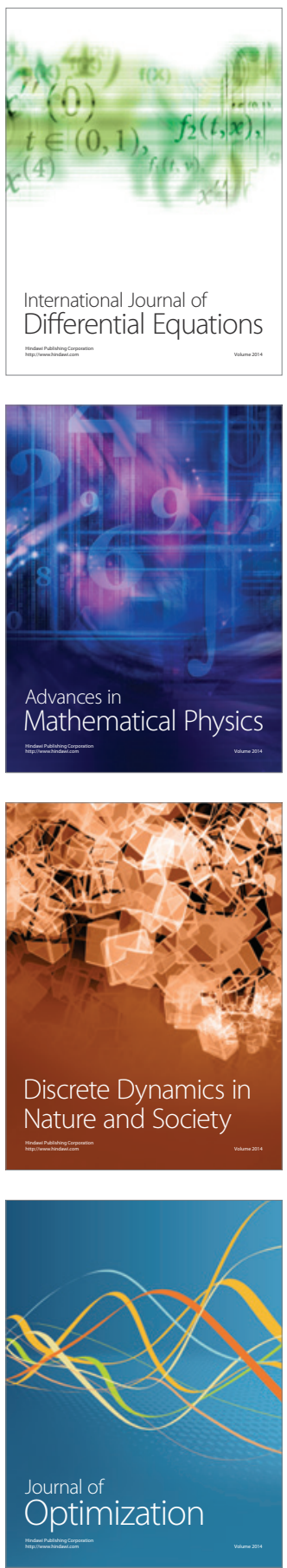